\title{
Design and anti-sway performance testing of pesticide tanks in spraying UAVs
}

\author{
Yu Zang ${ }^{1,2,3}$, Ying Zang ${ }^{1,2,3}$, Zhiyan Zhou ${ }^{1,2,3^{*}}$, Xiuyan $\mathrm{Gu}^{1,2,3}$, Rui Jiang ${ }^{1,2,3}$, Lingxi Kong ${ }^{1,2,3}$, \\ Xingang $\mathrm{He}^{1,2,3}$, Xiwen Luo ${ }^{1,2,3}$, Yubin Lan ${ }^{1,2,3}$ \\ (1. College of Engineering, South China Agricultural University / Guangdong Engineering Research Center for Agricultural Aviation \\ Application (ERCAAA), Guangzhou 510642, China; 2. National Joint Research Center for Precision Agricultural Aviation Application \\ Technology, Guangzhou 510642, China; 3. Key Laboratory of Key Technology on Agricultural Machine and Equipment (South China \\ Agricultural University), Ministry of Education, Guangzhou 510642, China)
}

\begin{abstract}
Anti-sway performance is one of the most important factors that affect the stability of unmanned aerial vehicles (UAVs). In this study, an anti-sway performance testing method and an evaluation formula for pesticide tanks were developed, which could facilitate the design and application of pesticide tank. The detection device mainly comprises a testing stand, a mounting and testing part, and a driving and control part. In the testing method, $P$ (maximum pressure) measured by a barometer and $t$ (fluctuation recovery time) measured by a high-speed camera were used as evaluation indexes. With the aim to determine the optimal position of the pressure detection device and the optimal filling ratio, four representative pesticide tanks were involved in this study. The results showed that the optimal position of the pressure detection device was at the middle position on the tank wall, halfway up from the bottom, and the optimal filling ratio was 0.8 . Using $P$ and $t$ as evaluation indexes, a comprehensive evaluation formula was developed based on these tests: $S=0.7 P_{i}+0.3 t_{i}$. Anti-sway performance of pesticide tanks was evaluated by a comprehensive score. A disk pesticide tank with a baffle was determined to be the best among the four types of pesticide tanks, showing a score of 1 . The scores of the other three pesticide tanks were $0.500,0.428$ and 0.612 , respectively. These results indicated that the baffle structure in the pesticide tank and the radian design of the tank wall can effectively improve the anti-sway performance of a pesticide tank. The proposed detection device and test method are simpler and more intuitive compared to other approaches in evaluating the anti-sway performance of pesticide tanks in spraying UAVs, and they can be used as a reference to guide the design of pesticide tanks and the stability evaluation of spraying UAVs.
\end{abstract}

Keywords: spraying UAV, pesticide tank, liquid sloshing, anti-sway performance test, detection method DOI: $10.25165 /$ j.ijabe. 20191201.4338

Citation: Zang Y, Zang Y, Zhou Z Y, Gu X Y, Jiang R, Kong L X, et al. Design and anti-sway performance testing of pesticide tanks in spraying UAVs. Int J Agric \& Biol Eng, 2019; 12(1): 10-16.

\section{Introduction}

With the development of agricultural aviation technology in China, the use of spraying unmanned aerial vehicles (UAVs) has increased over time. However, compared with some developed countries, agricultural aviation in China remains in the early stage ${ }^{[1]}$. With the popularization and wider application of UAVs for

Received date: $2018-04-20 \quad$ Accepted date: 2019-01-01

Biographies: Yu Zang, PhD candidate, research interest: agricultural aviation application, Email: 1024832959@qq.com; Ying Zang, PhD, Professor, research interests: agricultural mechanization and automation, Email: yingzang@ scau.edu.cn; Xiuyan Gu, Master, research interest: agricultural aviation application, Email: 1178931118@qq.com. Rui Jiang, PhD candidate, research interest: agricultural aviation application, Email: 469289264@qq.com; Lingxi Kong, Master candidate, research interest: agricultural aviation application, Email: 470299192@qq.com; Xingang He, Master candidate, research interests: agricultural aviation application, unmanned aviation vehicle performance testing. Email: 1096260969@qq.com; Xiwen Luo, Professor, research interests: agricultural equipment, precision agriculture, agricultural aviation application. Email: xwluo@scau.edu.cn; Yubin Lan, Professor, research interests: precision agriculture, agricultural aviation application. Email: ylan@scau.edu.cn.

*Corresponding author: Zhiyan Zhou, PhD, Professor, research interest: agricultural aviation application. College of Engineering, South China Agricultural University, Guangzhou 510642, China. Tel: +86-13560026139, Email: zyzhou@scau.edu.cn. pesticide spraying, problems regarding the safety and stability of UAVs are gradually emerging ${ }^{[2,3]}$. Liquid sloshing in the pesticide tank is one of the main factors affecting the stability of $\mathrm{UAV}^{[4]}$. Currently, most studies have focused on the distribution of droplet deposition ${ }^{[5,6]}$, control effect after spraying ${ }^{[7,8]}$, design of spraying systems $^{[9,10]}$, and remote sensing technology in relation to spraying $\mathrm{UAVs}^{[11,12]}$. However, only a few studies have investigated the anti-sway performance and evaluation of the pesticide tank. Most of them focused on optimization based on the appearance and structural design of the pesticide $\operatorname{tank}^{[13,14]}$. Moreover, no research on the detection devices and testing standards for the anti-sway performance of pesticide tanks in spraying UAVs was reported.

The pesticide tank is a key part of spraying UAV, and these pesticide tanks exhibit a great variety of shapes and structures. When a UAV needs to be stopped rapidly due to environmental factors (such as a gust of wind) or a head-turn during the spraying operation, the force in the flight direction and the vertical direction would be strengthened and produce a shock effect due to the inertia effect of the liquid ${ }^{[15]}$. The pressures in two directions would cause strong tensile or extrusion stress on the pesticide tank wall, which causes the entire aircraft to show harmful acceleration in these two directions ${ }^{[16]}$. To eliminate the harmful effects of acceleration and maintain the stability of the aircraft, the flight 
control unit needs to detect and respond these changes quickly. To deal with the shock effect, on the one hand, the flight control unit and the flight execution part of the whole aircraft need to be highly responsive, which not only increases the load of the components of the aircraft, but also increases the manufacturing cost. On the other hand, when the shock effect is beyond the adjustment range of the flight control unit, controlling the attitude of the aircraft becomes difficult, which may cause the UAV deviate from its intended route, show reduced work efficiency, or even crash $^{[17,18]}$. Therefore, technology to measure the anti-sway performance of the pesticide tank is very important for enhancing the safety and stability of spraying UAVs.

Liquid sloshing refers to the phenomenon in which liquid with a free interface moves in a limited space ${ }^{[19]}$. Liquid sloshing was mainly studied by theoretical analysis ${ }^{[20,21]}$, experimental analysis $^{[22,23]}$, computer simulation analysis ${ }^{[24,25]}$, etc. Such research has mainly focused on liquid sloshing in large pressure vessels, such as oil tankers ${ }^{[26,27]}$ and spacecraft ${ }^{[28,29]}$. This paper presents an anti-sway performance detection device to simulate the motion state and force characteristics of a pesticide tank in a UAV during spraying. Based on test results, an anti-sway performance detection method and an evaluation formula for pesticide tanks were developed, which could be used to guide the design and application of pesticide tanks.

\section{Design of the detection device}

Experimental studies on liquid sloshing have been mainly based on methods to simulate the actual force characteristics of oil tanks, fishing boat oil tanks, and spacecraft, such as scale-model experiments ${ }^{[30,31]}$, drop tower experiments ${ }^{[32,33]}$, and aircraft parabolic flight tests ${ }^{[34]}$. The proposed anti-sway performance detection device for pesticide tanks in spraying UAVs comprises a test table, a mounting and measuring part, a drive control part, and a transmission part, as shown in Figure 1.

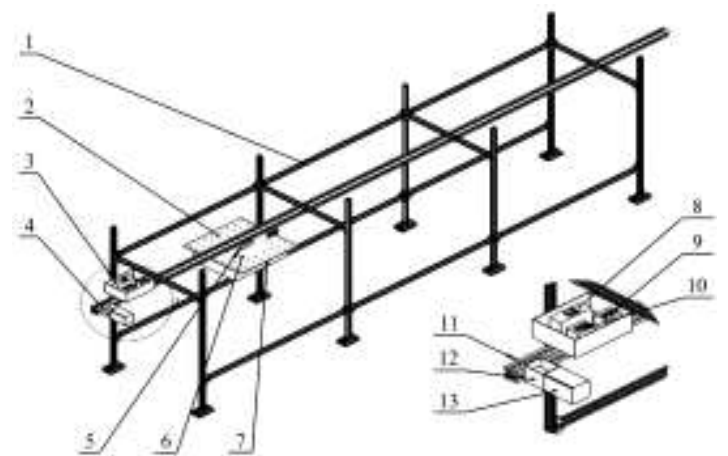

1. Test table 2. Mounting and measuring part 3. Drive control part 4. Transmission part 5. Slide 6. Connecting plate 7. Measured tank

8. Switching power supply 9. Servo driver 10. PLC controller 11. Synchronous belt 12. Coupling 13. Servo motor

Figure 1 Schematic of the anti-sway performance detection device for pesticide tanks in spraying UAVs

The test table is made up of an aluminum plate which is used for fixing the specimen to be tested and for support. The mounting and measuring part includes a connecting plate, a slider, a measured pesticide tank, and several sensors. A pesticide tank is fixed on the slider with the connecting plate so that the pesticide tank can be moved in a straight line on the slide rail. The drive control part includes a servo driver, a PLC controller, a switching power supply, and other components. The whole device adjusts the speed and displacement distance of the pesticide tank by adjusting the frequency output of the PLC controller. The transmission part is composed of a synchronous belt, a coupling, and a servo motor. The servo motor drives the synchronous belt by the coupling.

\section{Evaluation index and detection method}

In the process of UAV spraying, the greater the pressure of liquid sloshing, the greater the required control range of the flight control unit and the easier of the aircraft's yaw, crashing, etc. The longer the fluctuation recovery time after liquid sloshing, the longer is the impact time on the stability of the aircraft. Therefore, the maximum pressure and the fluctuation recovery time were selected as evaluation indexes of anti-sway performance to evaluate the effect of liquid sloshing on the safety and stability of the UAV.

\subsection{Maximum pressure detection}

The simplest and most direct method for measuring the pressure caused by liquid sloshing is to measure the pressure value on the pesticide tank wall. Common pressure sensors are large; while thin-film pressure sensors are small, but their water resistance performance is poor. It is difficult to fix these two types of sensors on the pesticide tank wall, and the pesticide tank might be damaged. To resolve this problem, we used an indirect measurement method in which the pressure difference is measured by pressure sensors. The pressure sensor is connected to the measuring point on the pesticide tank wall by a hollow tube. Liquid sloshing squeezes the air column, which changes the pressure; the measured pressure at that time is the relative pressure.

The measurement and installation methods are shown in Figure 2. The pressure-detection device is a closed box consisting of a barometer and PVC materials. It is connected to the measuring point by the hollow tube, and liquid sloshing squeezes the air column. At the beginning of the test, the barometer needs to be zeroed out to make sure the initial pressure difference is 0 . The measured pressure at this time is the relative pressure. We can evaluate the anti-sway performance of various pesticide tanks by comparing their maximum pressures.

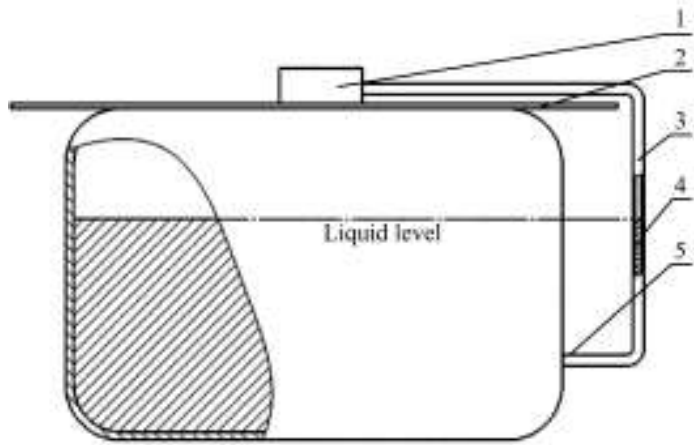

1. Pressure detection device 2 . Connecting plate 3 . Hollow tube 4. Liquid 5. Opening (measuring point)

Figure 2 Detection principle and installation method of the pressure detection device

\subsection{Fluctuation recovery time detection}

Fluctuation recovery time refers to the time needed for a liquid to move from a fluctuating state to a stationary state. In this work, a high-speed camera was used to record liquid fluctuation, and Equation (1) was used to calculate the fluctuation recovery time:

$$
t=\frac{n}{f}
$$

where, $t$ is the fluctuation recovery time; $n$ is the number of photographs taken by a high-speed camera during liquid fluctuation, and $f$ is the number of frames per second, fps. 


\subsection{Comprehensive evaluation formula}

Both the maximum pressure and the fluctuation recovery time affect the anti-sway performance of a pesticide tank in a spraying UAV; a single index is insufficient for assessment. Therefore, a comprehensive evaluation method is presented in this paper. At present, the main multi-index comprehensive evaluation includes 15 evaluation methods, such as the analytic hierarchy process weighted method ${ }^{[35]}$, artificial neural network method $^{[36]}$ and comprehensive index method ${ }^{[37]}$. The choice of weight coefficients includes 12 methods, such as expert consultation weights $^{[38]}$, factor analysis weights ${ }^{[39]}$, and independence weights ${ }^{[40]}$. According to the characteristics and requirements of the anti-sway performance testing, the comprehensive index method was employed as the comprehensive evaluation method, and the expert consultation weight method was used as the method of determining the weight coefficient in this paper.

The comprehensive index method is simple in principle and is easy to be operated ${ }^{[41]}$. The main operation steps are appropriate indicators selection, weights determination, formula establishment and calculation. Then, for comparison, index syntheses were performed according to the method of multiplication of similar indexes and the index of heterogeneous indexes to obtain the index $\mathrm{S}$ value. The calculation is as follows:

Index formula of high-level indicator:

$$
Y_{j}=\frac{X_{j}}{M_{j}}
$$

Index formula of low-optimal indicators:

$$
Y_{j}=\frac{M_{j}}{X_{j}}
$$

Final index calculation formula:

$$
S=\sum_{i=1}^{m} \prod_{j=1}^{n} Y_{i j}
$$

where, $X_{j}$ is the tested actual indicator value; $M_{j}$ is the selected standard indicator value, and $Y_{j}$ is the calculated index.

Therefore, during the process of comprehensive evaluation, the minimum value of the low-quality index is taken as the standard value in this paper. At the same time, the weights of the maximum pressure and the fluctuation recovery time obtained by the expert consultation weighting method are 0.3 and 0.7 , respectively. Combining Equations (2)-(4) with the weight coefficient, the evaluation index expressions of the three types are obtained as Equation (5).

$$
S=0.7 P_{i}+0.3 t_{i}
$$

\subsection{Test steps}

The steps in the testing process are briefly summarized as follows.

1) The pressure-detection device was installed on the pesticide tank for measuring. Then the pesticide tank was fixed on the slider with the connecting plate.

2) Liquid was added into the measured pesticide tank, and the barometer was adjusted to zero.

3) The computer, data transmitter, USB serial port, and data transmission receiver were connected to ensure the accurate data receiving.

4) The high-speed camera was turned on and focused on the pesticide tank, and the relevant parameters were adjusted.

5) The speed and distance were set by the PLC controller, and the servo motor was switched on. The data of the maximum pressure $\left(P_{1}\right)$ and the fluctuation recovery time $\left(t_{1}\right)$ were obtained.

6) Steps 2) and 3) were repeated, and the data were obtained as follows: $P_{1}, P_{2}, P_{3}, \ldots, P_{10}$, and $t_{1}, t_{2}, t_{3}, \ldots, t_{10}$;

7) The average of 10 groups of repeated tests was calculated. Then $\bar{P}$ and $\bar{t}$ was used to calculate the comprehensive evaluation formula to obtain $S$.

8) The test pesticide tank was replaced with another specimen, and the steps were repeated.

\section{Prototype and test}

\subsection{Anti-sway performance detection device}

\subsubsection{Prototype and control system}

Based on the schematic diagram of the anti-sway performance detection device (Figure 1), a prototype of a testing device was fabricated, as shown in Figure 3.

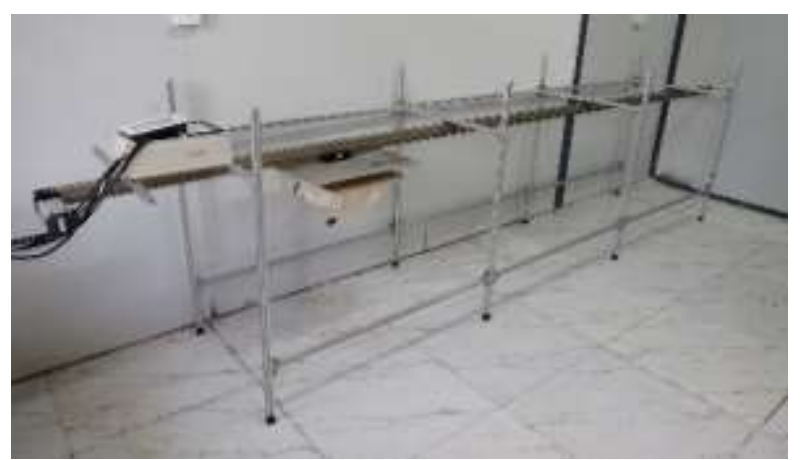

Figure 3 Prototype of the anti-sway performance detection device for pesticide tanks in spraying UAVs

During device operation, the PLC controller outputs pulse signals with various frequencies to the servo driver to control the operation of the servo motor. The servo motor is connected by the coupling to the sliding rail of the synchronous belt, and the mounting part of the pesticide tank is connected to the synchronous belt slider. Thus, the speed and displacement distance of the pesticide tank can be controlled by adjustment of the rotational speed and working time of the motor, as shown in Figure 4. The process of the pesticide tanks contains three continue phases as follows: accelerated motion, uniform motion, and braking deceleration.

The parameters of the device are shown in Table 1.

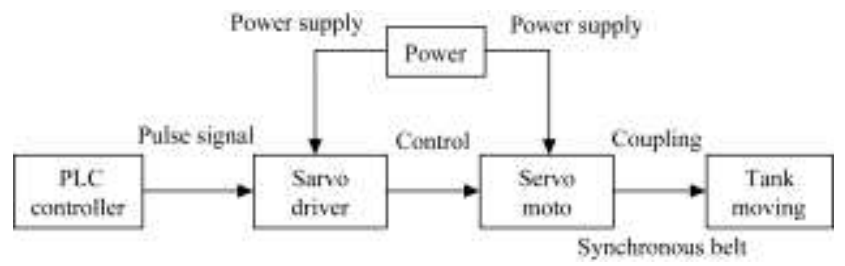

Figure 4 Process of controlling tank movement

Table 1 Parameters of the anti-sway performance detection device

\begin{tabular}{lc}
\multicolumn{1}{c}{ Parameter } & Specifics \\
\hline Length $/ \mathrm{m}$ & 4 \\
Servo motor & Panasonic MHMJ042G1U \\
Drive model & MBDKT2510E A5II \\
Power/W & 400 \\
Rotational speed $/ \mathrm{r} \cdot \mathrm{min}^{-1}$ & 5000 \\
Max load $/ \mathrm{kg}$ & 20 \\
Speed $/ \mathrm{m} \cdot \mathrm{s}^{-1}$ & $0-20\left(2 \mathrm{~m} \cdot \mathrm{s}^{-1}\right.$ is chosen $)$ \\
\hline
\end{tabular}

4.1.2 Maximum pressure detection device

The pressure on the pesticide tank wall caused by liquid sloshing is the most important factor that negatively affects the 
safety and stability of UAV operation. The smaller the maximum pressure is, the smaller the degree of interference with the flying attitude; hence, the better the anti-sway performance of the pesticide tank is. Therefore, a pressure detection device was introduced which was designed with a barometer and a hollow tube in this paper. Using the detection method and installation position shown in Figure 2, the pressure difference was measured by the barometer, and the pressure difference indicated the maximum pressure of the liquid. The pressure detection device mainly comprised a data transmitter, hollow pipe, barometer, gas tank, USB serial port, and data transmission receiver, as shown in Figure 5. The pressure measured by the barometer was transmitted to the computer by the data transmitter module and the USB serial port. Then the maximum pressure was recorded by the software.

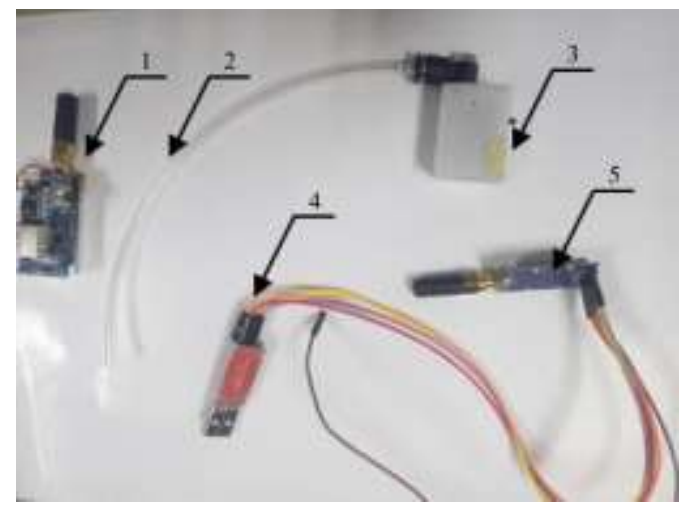

1. Data transmitter 2. Hollow tube 3. Barometer and gas tank 4. USB serial port 5. Data transmission receiver

Figure 5 Pressure detection device

\subsubsection{Detection device of fluctuation recovery time}

The high-speed camera used in this study was a Fastcam series model manufactured by the Huotulong (Shanghai) Company. It includes a mainframe engine, a camera, a light source, a transformer, an optical cable, and a power line. The maximum resolution is $1280 \times 1024$ pixels, the maximum frame rate is 210000 frames. 50 image frames were taken in each test in this study was, and the resolution was $1024 \times 1024$. Substituting Equation (6) the fluctuation recovery time was calculated as

$$
t=\frac{n}{50}
$$

where, $t$ is the fluctuation recovery time in seconds, and $\mathrm{n}$ is the number of photographs taken by the high-speed camera during liquid fluctuation.

Screenshots of test method and related software are shown in Figures 6 and 7.

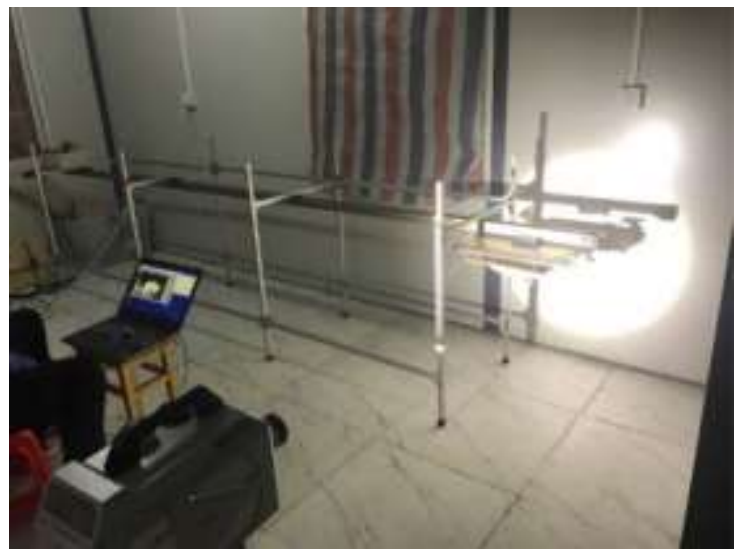

Figure 6 Fluctuation recovery time detection method

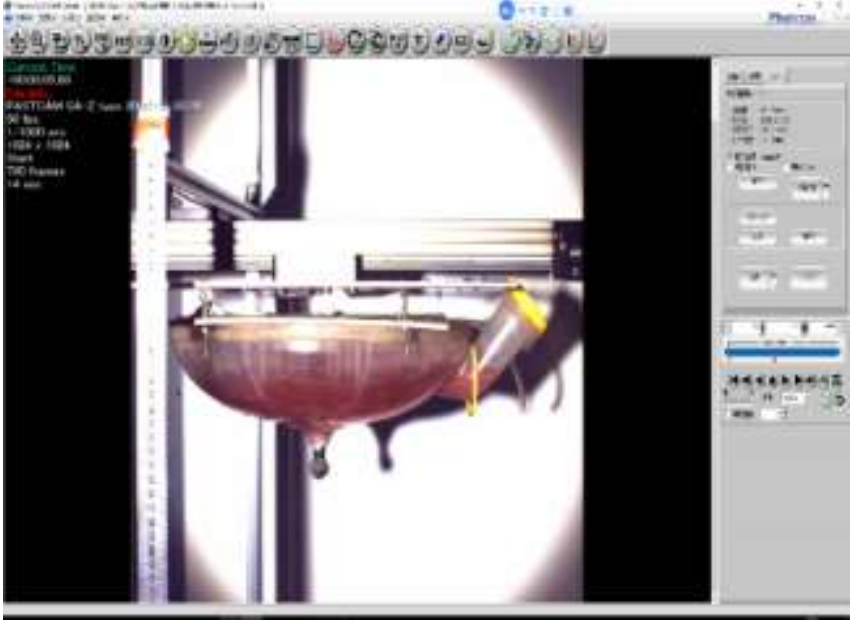

Figure 7 Software interface

\subsection{Measured pesticide tank of spraying UAV}

Because the levels of stress are different at different heights on the pesticide tank wall ${ }^{[42]}$, variation of the filling ratio also affects the fluctuation recovery time of the liquid. To select the best measurement position for the pressure detection device and the best filling ratio for fluctuation recovery time detection, four types of representative pesticide tanks were tested, as shown in Figure 8. The volume of all pesticide tanks is $8 \mathrm{~L}$.
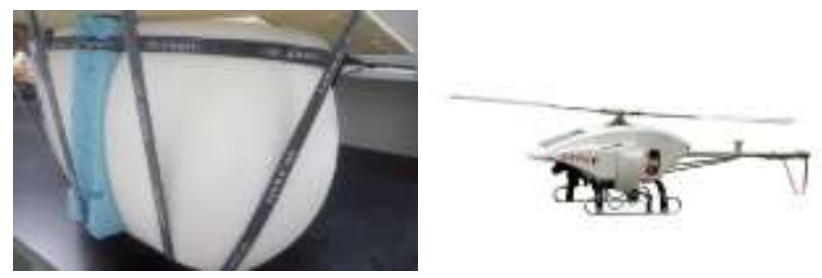

a. Double-column tank
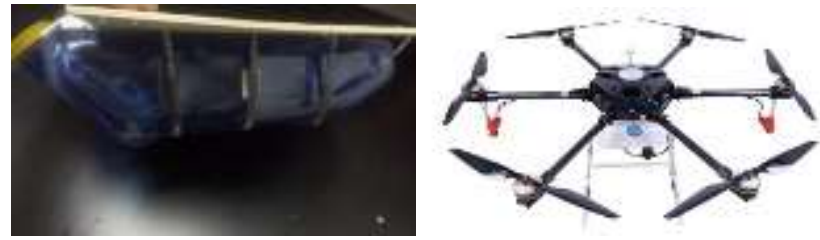

b. Rectangular tank
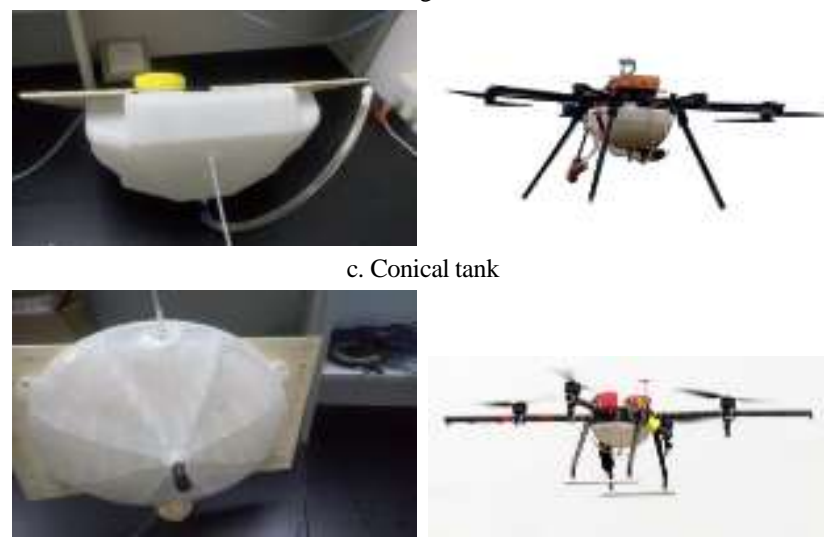

d. Disk pesticide tank with baffle

Figure 8 Four UAV pesticide tanks

4.3 Optimization of measurement position for the maximum pressure test

To explore the effect of the measurement position on the maximum pressure test, three measurement points were selected, they were at the bottom (1/3), middle (1/2), and top (2/3) of the pesticide tank, respectively. The travel speed of the pesticide tanks was $2 \mathrm{~m} / \mathrm{s}$, and the travel distance was $4 \mathrm{~m}$. Each pesticide 
tank was measured 10 times, and the average of 10 pressure values was calculated, as shown in Figure 9.

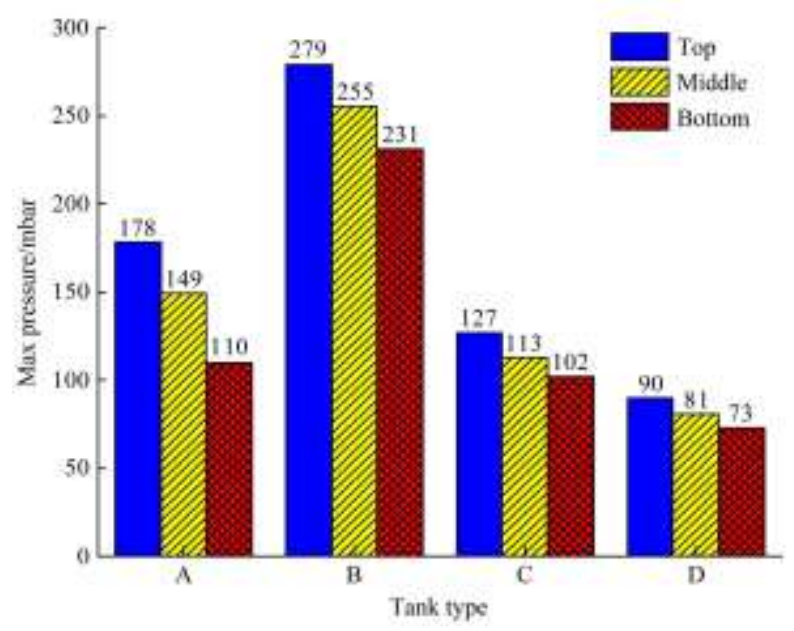

Figure 9 Maximum pressure at three measurement positions

To determine whether the results measured by the pressure detection device reflect the anti-sway performance of the considered pesticide tanks, the values for each pesticide tank were taken as samples. There was a total of four sample sets, and variance analysis were conducted by SPSS, as shown in Table 2 .

Table 2 Results of variance analysis for maximum pressure of four measured pesticide tanks

\begin{tabular}{cccccc}
\hline & Sum of Squares & df & Mean Square & $F$ & Sig. \\
\hline Between Groups & 256225.650 & 3 & 85408.550 & 236.606 & .000 \\
Within Groups & 20214.533 & 56 & 360.974 & & \\
Total & 276440.183 & 59 & & & \\
\hline
\end{tabular}

As seen in Table 2, sig $=0.000<0.01$; therefore, there were significant differences between the pressures measured for the four types of pesticide tanks. Thus, the results indicated that pressure measured by the pressure detection device can be used as an evaluation index of anti-sway performance of pesticide tanks.

To select the best measurement position, variance analysis of the detection results obtained for the four types of pesticide tanks at three measuring positions was conducted by SPSS. The best position for pressure measurement was determined by comparing $F$ values. The bigger the $F$ value was, the greater the differences between the results measured for the four types of pesticide tanks, as shown in Table 3.

Table 3 Results of variance analysis for maximum pressure at three measuring positions

\begin{tabular}{ccc}
\hline Measuring positions & Sig. & $F$ \\
\hline Top & 0.000 & 5722.293 \\
Middle & 0.000 & 6060.896 \\
Bottom & 0.000 & 2013.859 \\
\hline
\end{tabular}

The variance analysis showed the following relationship:

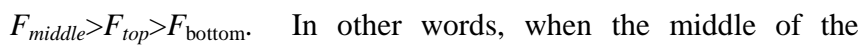
pesticide tank wall was selected as the measurement position, the measured results showed significant differences. Therefore, in order to evaluate the anti-sway performance of pesticide tanks in spraying UAVs better, the sensor should be placed at the middle of the pesticide tank wall before testing.

\subsection{Optimization of the filling ratio for fluctuation recovery} time testing

To explore the impact of the filling ratio on the fluctuation recovery time test, filling ratios of $0.4,0.6$ and 0.8 were set up.
The travel speed of the pesticide tank was $2 \mathrm{~m} / \mathrm{s}$, and the travel distance was $4 \mathrm{~m}$. Each pesticide tank was measured 10 times, and the average of 10 results was calculated as the fluctuation recovery time, as shown in Figure 10.

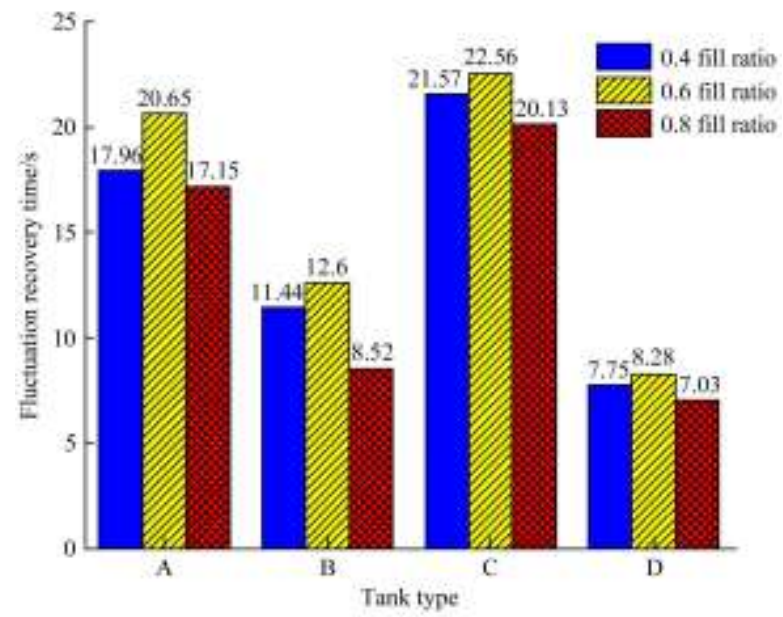

Figure 10 Fluctuation recovery time of various filling ratios

To determine whether the fluctuation recovery time measured by the high-speed camera can distinguish the anti-sway performance of various pesticide tanks, the values for each pesticide tank were taken as a sample. There was a total of four sample sets, and variance analysis was conducted by SPSS, as shown in Table 4.

Table 4 Results of variance analysis of fluctuation recovery time for four measured pesticide tanks

\begin{tabular}{cccccc}
\hline & Sum of Squares & df & Mean Square & $F$ & Sig. \\
\hline Between Groups & 1863.654 & 3 & 621.218 & 338.099 & 0.000 \\
Within Groups & 102.894 & 56 & 1.837 & & \\
Total & 1966.547 & 59 & & & \\
\hline
\end{tabular}

As seen in Table 4, sig $=0.000<0.01$; therefore, there were significant differences between the fluctuation recovery times for the four kinds of pesticide tanks. Thus, the results demonstrate that the fluctuation recovery time measured by the high-speed camera can be used as an evaluation index of the anti-sway performance of pesticide tanks.

To optimize the filling ratio, variance analysis of the detection result obtained for the four kinds of pesticide tanks with three different filling ratios were conducted by SPSS. The best filling ratio was selected by comparing $t$ values, as shown in Table 5 .

Table 5 Results of variance analysis for fluctuation recovery time at three measuring positions

\begin{tabular}{ccc}
\hline Filling ratio & Sig. & $F$ \\
\hline 0.4 & .000 & 1279.059 \\
0.6 & .000 & 1258.007 \\
0.8 & .000 & 3049.685 \\
\hline
\end{tabular}

The variance analysis results showed the following relationship: $t_{0.8}>t_{0.4}>t_{0.6}$. In other words, when the filling ratio was 0.8 , the fluctuation recovery time of the four kinds of pesticide tanks showed significant differences. Therefore, to evaluate the anti-sway performance of pesticide tanks in spraying UAVs better, the filling ratio should be 0.8 before testing.

\subsection{Test results of anti-sway performance of four pesticide tanks}

The above experiments and analyses demonstrated that the maximum pressure measured at the middle position and the 
fluctuation recovery time can be used as evaluation indexes of the anti-sway performance of pesticide tanks. The test results obtained for the four kinds of pesticide tanks are shown in Table 6 .

Table 6 Liquid sloshing test results obtained for four kinds of UAV pesticide tanks

\begin{tabular}{ccc}
\hline Tank type & Max pressure/mbar & Fluctuation recovery time/s \\
\hline A & 149 & 17.96 \\
B & 255 & 11.44 \\
C & 113 & 21.57 \\
D & 81 & 7.75 \\
\hline
\end{tabular}

1) It can be observed that $P_{B}>P_{A}>P_{C}>P_{D}$ from Table 6 . That is, considering the effect of liquid sloshing on the stability of UAV, pesticide tank $\mathrm{D}$ showed the best anti-sway performance, and pesticide tank B showed the worst.

One possible explanation is that the shape of pesticide tank B is the longest of the four types of tanks. It has a greater speed when the liquid in the rear reaches the front of the pesticide tank wall. Moreover, there is no radian treatment on the pesticide tank wall. Also, it has a larger accumulated liquid volume when the pesticide tank tilts in one direction. Therefore, the pressure produced by the liquid sloshing is the greatest. However, in pesticide tank $\mathrm{D}$, the baffle structure in the pesticide tank is larger, and radian treatment is conducted on the pesticide tank wall, so the pressure produced by the liquid sloshing is the smallest.

2) It can be seen that $t_{C}>t_{A}>t_{B}>t_{D}$ from Table 6 . Considering the effect of action time of liquid sloshing on the stability of UAV, pesticide tank D showed the best anti-sway performance, whereas pesticide tank $\mathrm{C}$ showed the worst.

A possible explanation is that pesticide tank B is the longest. Therefore, the effect of the sloshing is reduced when the liquid in the pesticide tank hits the front wall of the tank and then dashes onto the liquid from behind the wall of the tank. However, pesticide tank $\mathrm{D}$, which has a baffle structure and curving walls, can eliminate some of the sloshing effect.

A comprehensive evaluation formula of pesticide tanks was developed to compare the anti-sway performance of various kinds of pesticide tanks more conveniently. The comprehensive evaluation forms are shown in Table 7 based on the Equation (5) and Table 6.

Table 7 Comprehensive evaluation form

\begin{tabular}{|c|c|c|c|c|c|}
\hline \multirow{2}{*}{$\begin{array}{l}\text { Tank } \\
\text { type }\end{array}$} & \multicolumn{2}{|c|}{ Max pressure } & \multicolumn{2}{|c|}{ Fluctuation recovery time } & \multirow{2}{*}{$\begin{array}{l}\text { Total } \\
\text { score }\end{array}$} \\
\hline & Indexed result & Weight ratio & Indexed result & Weight ratio & \\
\hline A & 0.53 & \multirow{3}{*}{0.7} & 0.43 & \multirow{4}{*}{0.3} & 0.5 \\
\hline B & 0.32 & & 0.68 & & 0.428 \\
\hline $\mathrm{C}$ & 0.72 & & 0.36 & & 0.612 \\
\hline $\mathrm{D}$ & 1 & & 1 & & 1 \\
\hline
\end{tabular}

\section{Conclusions}

Based on the stress characteristics of pesticide tanks in spraying UAVs, the design of a detection device was presented in this paper for anti-sway performance testing of pesticide tanks. The test conclusions can be summarized as follows.

1) The best measurement position to assess anti-sway performance of a pesticide tank was determined. The pressure difference at the middle position of the pesticide tank wall was measured by the pressure detection device, and the pressure caused by liquid sloshing in various pesticide tanks could be compared. At this position, the results showed significant differences. The maximum pressure measured at the middle position can be used as an evaluation index of anti-sway performance of pesticide tanks.

2) The best fill ratio to assess the anti-sway performance of a pesticide tank was also determined. The fluctuation recovery time of the liquid in a pesticide tank with the filling ratio is 0.8 was measured by a high-speed camera. With this filling ratio, the fluctuation recovery time of the liquid caused by sloshing in various pesticide tanks could be compared. With this filling ratio, the results showed significant differences. The fluctuation recovery time can be used as an evaluation index of the anti-sway performance of pesticide tanks.

3 ) The stability of four kinds of representative pesticide tanks was compared in this study. The anti-sway performance of the disk pesticide tank with a baffle was the best. Its minimum pressure was $81 \mathrm{mbar}$, and it had the shortest fluctuation recovery time of $7.75 \mathrm{~s}$. This may be attributed to the fact that increasing the baffle structure in the pesticide tank and radian treatment on the pesticide tank wall effectively improves the anti-sway performance of pesticide tank. However, the length of a pesticide tank also influences its anti-sway performance. As the length increases, the pressure increases, but the fluctuation recovery time is reduced. Therefore, in order to select the proper length, further tests are required.

4) The following comprehensive formula for the anti-sway performance evaluation of pesticide tanks was proposed in this paper: $S=0.7 P_{i}+0.3 t_{i}$. The score of the disk pesticide tank with a baffle was 1. This was the highest score among all tanks considered in this study, and it indicates excellent anti-sway performance. Comprehensive scores calculated by the proposed formula can be an important basis to evaluate the anti-sway performance of pesticide tanks in spraying UAVs. It provides a theoretical basis for the evaluation of their safety and stability performance.

In comparison with simulation analysis, the anti-sway performance of pesticide tanks can be evaluated more simply and intuitively by the method presented in this paper. The proposed detection device has the advantages of a simple mechanical structure, convenient installation, low cost, and strong applicability. However, only four typical types of pesticide tanks were considered in this study. Another limitation of this study was the lack of variety of pesticide tank materials and internal fillings in the pesticide tank. Therefore, further experimental study is required.

\section{Acknowledgements}

Authors wish to thank the Science and Technology Plan of Guangzhou and Guangdong Province of China (Grant No. 2015B020206003, 201807010111, 2017B090907031, 2017B0909030 07) and Innovative Research Team of Guangdong Province Agriculture Research System (2017LM2153) for funding this research. We also thank the anonymous reviewers for their critical comments and suggestions to improve the manuscript.

\section{[References]}

[1] Zhou Z Y, Ming R, Zang Y, He X G, Luo X W, Lan Y B. Development status and countermeasures of agricultural aviation in China. Transactions of the CSAE, 2017; 33(20): 1-20. (in Chinese)

[2] Zang D Y, Lan Y B, Chen L P, Wang X, Liang D. Current status and future trends of agricultural aerial spraying technology in China. Transactions of the CSAM, 2014; 45(10): 53-59. (in Chinese).

[3] Zang Y, Gu X Y, Zhou Z Y, Luo X W, Zang Y, Qi X Y, et al. Review of tensairity and its applications in agricultural aviation. Int J Agric \& Biol Eng, 2016; 9(3): 1-14. 
[4] Li X, Zhang J X, Qu F, Zhang W Q, Wang D S, Li W. Optimal design of anti-sway inner cavity structure of agricultural UAV pesticide tank. Transactions of the CSAE, 2017; 33(18): 72-79. (in Chinese)

[5] Wang L, Lan Y B, Hoffmann W C, Bradley K F, Chen D, Wang S M. Design of variable spraying system and influencing factors on droplets deposition of small UAV. Transactions of the CSAM, 2016; 47(1): 15-22. (in Chinese)

[6] Chen S D, Lan Y B, Bradley K F, Li J Y, Liu A M, Mao Y D. Effect of wind field below rotor on distribution of aerial spraying droplet deposition by using multi-rotor UAV. Transactions of the CSAM, 2017; 48(8): 105-113. (in Chinese)

[7] Qin W C, Qiu B J, Xue X Y, Chen C, Xu Z F, Zhou Q Q. Droplet deposition and control effect of insecticides sprayed with an unmanned aerial vehicle against plant hoppers. Crop Protection, 2016; 85: 79-88.

[8] Gao Y Y, Zhang Y T, Zhao Y C, Li X H, Yang D B, Yuan H Z. Primary studies on spray droplet distribution and control effects of aerial spraying using unmanned aerial vehicle (UAV) against the corn borer. Plant Protection, 2013; 39(2): 152-157.

[9] Liu W L, Zhou Z Y, Chen S D, Luo X W, Lan Y B. Status of aerial electrostatic spraying technology and its application in plant protection UAV. Journal of Agricultural Mechanization Research, 2018; 5: 1-9. (in Chinese)

[10] Jiang R, Zhou Z Y, Xu Y, Lan Y B, Luo X W. Design and experiment of liquid quantity monitor for pesticide tank in spraying UAV. Transactions of the CSAE, 2015; 31(8): 42-47. (in Chinese)

[11] Xu T Y, Yu F H, Cao Y L, Du W, Ma M Y. Vertical distribution of spray droplet deposition of plant protection multi rotor UAV for japonica rice. Transactions of the CSAM, 2017; 48(10): 101-107. (in Chinese)

[12] Yu F H, Xu T Y, Du W, Ma H, Zhang G S, Chen C L. Radiative transfer models (RTMs) for field phenotyping inversion of rice based on UAV hyperspectral remote sensing. Int J Agric \& Biol Eng, 2017; 10(4): $150-157$

[13] He Y, Xiao Y Z. An anti-sway pesticide tank of plant protection UAV: CN201520086118.0. 2015-8-5. (in Chinese)

[14] Shao X J, Han X, Wang N. A pesticide tank of plant protection UAV with internal curve surface: CN201620934225.9. 2017-1-4. (in Chinese)

[15] Deng M, Yue B Z, Huang H. Study on the equivalent mechanical model for large amplitude slosh. Journal of Astronautics, 2016; 37(6): 631-638. (in Chinese).

[16] Yang Y, Jing W, Kang Z. An equivalent mechanical model for Liquid sloshing on spacecraft. Journal of Aeronautics Astronautics \& Aviation, 2016; 48(2): 75-81.

[17] Pletcher R H, Chen K H, Kelecy F J. Numerical and experimental study of three-dimensional liquid sloshing flows. Journal of Thermophysics \& Heat Transfer, 2015; 8(3): 507-513.

[18] Yin S Y, Wang S F, Chen C. Research on method for determination of UAV safety index requirements. Modern Defence Technology, 2015; 43(2): 63-67. (in Chinese)

[19] Hu Q, Li Y, Liu J T, Liang J Q. Research on liquid sloshing performance in vane type tank under microgravity. IOP Conference Series-Materials Science and Engineering, 2016; 129: 012016.

[20] Haroun M A, Chen W. Large amplitude liquid sloshing in seismically excited tanks. Earthquake Engineering \& Structural Dynamics, 2015; 25(7): 653-669

[21] Wei C, Wang L, Shabana A A. A total Lagrangian ANCF liquid sloshing approach for multibody system applications. Journal of Computational \& Nonlinear Dynamics, 2015; 10(5): 51-54.

[22] Yan G R, Rakheja S, Siddiqui K. Analysis of transient fluid slosh in partly-filled tanks with and without baffles: Part 1-model validation. International Journal of Heavy Systems, 2010; 17(3-4): 359-379.
[23] Hu Q, Li Y, Yao C, Liu J T. Experiment of liquid sloshing performance in bulky propellant tank. Aerospace Control and Application, 2016; 42(3): 44-48. (in Chinese)

[24] Singal V, Bajaj J, Awalgaonkar N, Tibdewal S. CFD analysis of a kerosene fuel tank to reduce liquid sloshing. In Katalinic B. Ed. Procedia Engineering, Elsevier, 2015; Vol.100, pp.1732.

[25] Kang H, Xu J, Zhu J H, Tong M B. Study of liquid sloshing in missile fuel tank based on SPH method. Wireless Internet Technology, 2015; 3 : 76-79.

[26] $\mathrm{Li} \mathrm{S} \mathrm{J.} \mathrm{Finite} \mathrm{element} \mathrm{analysis} \mathrm{for} \mathrm{cycling} \mathrm{tanker.} \mathrm{Guangzhou:}$ Guangdong University of Technology, 2011. (in Chinese)

[27] Zuo Y G, Chen X. Research on effect of vehicle cross-section shape on roll Stability. Journal of Chongqing University of Technology: Natural Science, 2018; 32(8): 47-51. (in Chinese)

[28] Kolaei A, Rakheja S, Richard, M J. Effects of tank cross-section on dynamic fluid slosh loads and roll stability of a partly-filled tank truck. European Journal of Mechanics B-fluids, 2018; 46: 46-58.

[29] Deng M L, Yue B Z. Attitude dynamics and control of liquid filled spacecraft with large amplitude fuel slosh. Journal of Mechanics, 2018; 33(1): 125-136.

[30] Zhu Z Y. Study on sloshing effect of mass transfer between vapor and liquid phases in oil tank of fishing boats. Zhejiang: Zhejiang Ocean University, 2014. (in Chinese)

[31] Zhu Z. Study on vehicle-liquid coupling dynamic characteristics anti-rollover control method for tank vehicles. Jilin University, 2018. (in Chinese)

[32] Berry R L, Tegart J R. Experimental study of transient liquid motion in orbiting spacecraft. NASA Sti/recon Technical Report N, 1975; 76.

[33] Himeno T, Watanabe T, Konno A. Numerical analysis for propellant management in rocket tanks. Journal of Propulsion \& Power, 2012; 21(1) $76-86$.

[34] Huang H D. The research of liquid sloshing under low gravity environment. Journal of Astronautics, 1980; 1: 76-89. (in Chinese)

[35] Krejci J, Stoklasa J. Aggregation in the analytic hierarchy process: Why weighted geometric mean should be used instead of weighted arithmetic mean. Expert Systems with Applications, 2018; 114: 643-97-106.

[36] Brar L S, Elsayed K. Analysis and optimization of cyclone separators with eccentric vortex finders using large eddy simulation and artificial neural network. Separation and Purification Technology, 2018; 269-283.

[37] Shan M Q, Qian Y, Yu S, Guo S C, Zhang L, Ding A W, et al. Anti-inflammatory effect of volatile oil from Schizonepeta tenuifolia on carrageenin-induced pleurisy in rats and its application to study of appropriate harvesting time coupled with multi-attribute comprehensive index method. Journal of Ethnopharmacology, 2018; 194: 580-586.

[38] Bracke M B M, Zonderland J J, Bleumer E J B, Wu S. Expert consultation on weighting factors of criteria for assessing environmental enrichment materials for pigs. Applied Animal Behaviour Science, 2007; 104(1-2): 14-23.

[39] Leng Y, Chen Y, Fu Q, Chen Z. Constructing empowerment method based on index independence. Statistics \& Decision, 2016; 19: 9-11. (in Chinese)

[40] Hui W, Li C, Chen K, Xue M Q, Liang Q. Multi-index comprehensive evaluation method and choice of weight coefficient. Journal of Guangdong College of Pharmacy, 2007; 23(5): 583-589. (in Chinese)

[41] Mir I, Maqsood A, Akhtar S. Optimization of dynamic soaring maneuvers to enhance endurance of a versatile UAV. IOP Conference Series-Materials Science and Engineering, 2017.

[42] Wang X, Dai N Q, Liu S Q, Zhang Z L, Dai R J. Structure analysis of semi liquid tanker semitrailer based on fluid-solid interaction. Auto Time, 2016; 4: 37-38. 